\title{
INTERSTELLAR LINES OTHER THAN HYDROGEN
}

\author{
G. H. HER BIG
}

Lick Observatory, University of California, Santa Cruz, Calif., U.S.A.

I would like to extrapolate beyond the present state of interstellar line spectroscopy toward the next generation of satellite-borne equipment, but with due recognition of the fact that more work still has to be done from the Earth's surface.

Experience with narrow interstellar absorption lines shows that the faintest detectable lines have equivalent widths of about 0.1 times the resolution. Since the best farultraviolet spectrograms now available have a resolution of about $1 \AA$, so the detection limit is about $100 \mathrm{~m} \AA$. Most of what I have to say is directed toward the advantages and problems of the era when lines having one-tenth of this strength are observable from outside the atmosphere.

One of the most central problems of this subject is that of the chemical composition of the interstellar gas, and the possibility that it varies from place to place in the interstellar medium. From the Earth's surface, the total abundance of an element in the gas has to be inferred from the abundance of one or two of its ions. This calculation involves the competition between the ionization rate (a radiation field and an energeticparticle spectrum operating upon wavelength-dependent photoionization and collisional-ionization probabilities) and the recombination rate (dependent upon the electron-capture cross-sections for all the levels, and on the electron temperature). Unfortunately, these rates have to be multiplied by the local concentrations of the various species, $n(r)$ (in $\mathrm{cm}^{-3}$ ), while the observations give the total number of ions in the column between Earth and star, $N\left(\right.$ in $\left.\mathrm{cm}^{-2}\right)=\int n(r) \mathrm{d} r$. Therefore, to correct for ionization one has either to make some assumption about the distribution of matter along the line of sight, or to minimize the effect by some manipulation of the data.

There is evidence from terrestrial observations, interpreted in this way, that the interstellar abundances of $\mathrm{Na}$ and $\mathrm{K}$ are approximately 'cosmic' with respect to atomic $\mathrm{H}$, while $\mathrm{Ca}$ and $\mathrm{Ti}$ are abnormally low. Upper limits that are not in conflict with chondritic data can be set on $\mathrm{Li}$ and $\mathrm{Be}$ (Herbig, 1968). But the results are no stronger than the weakest link in the rather extended calculations that lead to the corrections for ionization.

Surely one of the most attractive prospects of space spectroscopy is that it will provide direct information on more ions so that, in some elements, column abundances can be obtained directly without these very difficult allowances for ionization. The elements listed in Table I are particularly favorable in this respect. Listed also in the table are the wavelengths of the $\mathrm{K}$ absorption edges for these same elements, as a reminder that there is in principle a means of determining the total column abundance of an element that does not involve the state of ionization or whether the atom is free or bound, although information on the chemical association of the atom may be contained in the fine structure of the edge. But the result, however accurate or however 
obtained, is still $N$, and the determination of the local concentration $n(r)$ must depend upon knowledge of the radiation field, upon the availability of the necessary laboratory data, and upon some model for the intervening interstellar medium. Let us consider these last two problems in more detail.

\section{TABLE I}

\begin{tabular}{|c|c|c|c|}
\hline \multicolumn{4}{|c|}{$\begin{array}{l}\text { Abundant atoms having ions with } \\
\text { resonance lines } \lambda>911 \AA\end{array}$} \\
\hline Ion & $\begin{array}{l}\text { Resonance } \\
\text { Line }\end{array}$ & Ion & $\begin{array}{l}\text { Resonance } \\
\text { Line }\end{array}$ \\
\hline $\mathrm{CI}_{\mathrm{I}}$ & $1656 \AA$ & $\mathrm{N}_{\mathrm{I}}$ & $1200 \AA$ \\
\hline CII & 1334 & $\mathrm{~N}_{\text {II }}$ & 1083 \\
\hline CIII & 977 & $\mathrm{~N}_{\text {III }}$ & 989 \\
\hline CIV & 1549 & (NIV & 765) \\
\hline$K$ edge $C$ & 43.77 & $\begin{array}{l}\mathrm{N} v \\
\mathrm{~K} \text { edge } \mathrm{N}\end{array}$ & $\begin{array}{l}1240 \\
31.05\end{array}$ \\
\hline AlI & 3944 & Si I & 2514 \\
\hline AlII & 1670 & SiII & 1808 \\
\hline $\mathrm{Al}_{\text {III }}$ & 1854 & Si III & 1206 \\
\hline $\mathrm{K}$ edge $\mathrm{Al}$ & 7.95 & $\begin{array}{l}\text { Silv } \\
\text { K edge Si }\end{array}$ & $\begin{array}{l}1393 \\
6.74\end{array}$ \\
\hline PI & 1774 & $\mathbf{S}_{\mathbf{I}}$ & 1807 \\
\hline PII & 1532 & SII $_{\text {II }}$ & 1259 \\
\hline PIII & 1334 & SIII & 1190 \\
\hline PIV & 950 & Siv & 1062 \\
\hline$K$ edge $P$ & 5.79 & K edge S & 5.02 \\
\hline
\end{tabular}

A pressing need exists for more detailed information on absolute photo-ionization cross-sections as a function of wavelength, from threshold to about $504 \AA$ (below which there is ordinarily too little stellar radiation to contribute significantly). The experimental data are most complete for the ground states of the alkali metals (LiI, $\mathrm{NaI}, \mathrm{K}_{\mathrm{I}}$ ) and for CaI, but there is urgent need for more work, especially on $\mathrm{MgI}$ and Al I. But special priority should be attached to studies of the atoms listed in Table I. For ionized atoms, however, the technical limitations of absorption-tube work are such that photoionization probabilities for ions and for excited states will apparently have to come from theory. The laboratory absorption data include the autoionization structure if any, so the photoionization rate includes the contribution of autoionization. But the contribution of dielectronic recombination to the recombination rate under interstellar conditions has not been taken into account.

The increasing strength of interstellar lines with distance means that the line of sight is passing through an increasing number of regions with increasingly heterogeneous properties. The problem of recovering $n$ from $N$ thus becomes progressively more difficult. It seems obvious that more will be learned from a concentrated attack upon the interstellar spectra of a few, relatively nearby stars in which the line structure 
is not complex, and for which some independent estimate can be made of the radiation field and the density profile in the line of sight. Therefore I wish to recommend for particular attention by optical, radio, and ultraviolet observers the four stars listed in Table II. These stars show especially simple line structure in Hobbs' (1969) atlas of high-resolution $\mathrm{Na}$ I profiles.

TABLE II

Stars recommended for detailed attention

\begin{tabular}{llcrl} 
Star & \multicolumn{1}{l}{$b^{\text {II }}$} & Spectral type & $E(\mathrm{~B}-\mathrm{V})$ & $N(\mathrm{Na} \mathrm{I})$ \\
& & & & \\
$\delta$ Cyg AB & $+10^{\circ}$ & B9.5 III & 0.00 & $2 \times 10^{11} \mathrm{~cm}^{-2}$ \\
$\delta$ Sco & +22 & B0 V & +0.19 & $42 \times 10^{11}$ \\
$o$ Per & -18 & B1 III & +0.32 & - \\
$\zeta$ Oph & +24 & O9.5 V & +0.32 & $490 \times 10^{11}$
\end{tabular}

The long-standing $\mathrm{Ca} / \mathrm{Na}$ abundance anomaly is a good example of the abundance problems that await solution. The apparent deficiency of $\mathrm{Ca}$ in the interstellar gas is still unresolved, despite recurrent optimism that improvement of some aspect of the ionization correction would provide an explanation. The amount of the Ca deficiency with respect to $\mathrm{Na}$ seems to vary in different directions (e.g., it is particularly large in front of $\zeta \mathrm{Oph}$ ), but one must be cautious with $N(\mathrm{Ca}$ II $)$ values determined from $\mathrm{K} / \mathrm{H}$ ratios, which are susceptible to serious systematic error. The next members of the $4^{2} \mathrm{~S}-\mathrm{n}^{2} \mathrm{P}^{\circ}$ series of $\mathrm{Ca}$ II lie at $1649,1652 \AA(n=5)$ and $1341,1342 \AA(n=6)$. High priority should be given with space spectrographs to the determination of accurate equivalent widths for these higher members in a few interstellar spectra*, to be sure at least that the curve-of-growth formalism is correct. This same problem will probably arise when observations become available of the interstellar lines of $\mathrm{Mg}_{\mathrm{II}}$ at 2795 , $2802 \AA$. In the case of $\mathrm{NaI}$, the second members of the series, at $3302 \AA$, fortunately are accessible from the Earth's surface.

If this apparent underabundance of $\mathrm{Ca}$ in the gas persists, then the following more interesting hypotheses will have to be considered as possible explanations:

(1) A superionization phenomenon: the missing $\mathrm{Ca} I$ and $\mathrm{Ca}$ II may have been transferred to CaIII. This will be a difficult matter to confirm even from space, since the strongest resonance lines of Ca III lie at $490 \AA$ and shortward (Borgström, 1968).

(2) A chemical phenomenon: the missing $\mathrm{Ca}$ may have been bound up in the interstellar dust. This would be quite understandable in the context of the recent hypothesis that the interstellar particles are composed of refractory $\mathrm{Mg}, \mathrm{Al}$, and $\mathrm{Ca}$ silicates plus metallic iron (Larimer, 1967; Herbig, 1969). One would then expect that the concentrations of free $\mathrm{Mg}, \mathrm{Al}$, etc. in the gas would also show the effects of such selective depletion.

* One expects these lines to be weak, because $\mathrm{Ca}$ II is isoelectronic with $\mathrm{KI}$, in which the $f$-values of the higher series members are very small. 
(3) A molecular process: the missing $\mathrm{Ca}$ may have been tied up in some tightly bound, spectroscopically undetectable molecule in the gas. A candidate might be $\mathrm{CaC}$, whose spectrum has not yet been observed in the laboratory.

Whatever the mechanism, it is clear that $\mathrm{Ca}$ must be present in the interstellar medium in some form and in approximately the solar or chondritic proportions, as demonstrated by the $\mathrm{Ca}$ contents of stars formed from that same interstellar material (although there is a hint that the $\mathrm{Ca} / \mathrm{H}$ ratio may vary slightly with age; Spite, 1968).

\section{References}

Borgström, A.: 1968, Ark. Fys. 38, 243.

Herbig, G. H.: 1968, Z. Astrophys. 68, 243.

Herbig, G. H.: 1969, 'Pre-Main Sequence Stellar Evolution', Mem. Soc. Roy. Sci. Liège (in press). Hobbs, L. M.: 1969, Astrophys. J. 157, 135.

Larimer, J. W.: 1967, Geochim. Cosmochim. Acta 31, 1215.

Spite, M.: 1968, Ann. Astrophys. 31, 269.

\section{Discussion}

Spitzer: There is one central feature relevant to the sodium-calcium ratio which was pointed out years ago and which should be stressed again here. Observations indicate that for the high-velocity components of interstellar lines the ratio of sodium atoms to calcium atoms appears to be nearly normal.

If the low calcium-sodium ratio in normal clouds is attributed to the calcium being locked up either in grains or in molecules, than one must find some mechanism by which the calcium can be released back into the gas as atoms when the interstellar medium is being accelerated. Quite possibly acceleration of clouds by newly born $O$ stars or by supernova explosions can provide such a mechanism.

Wickramasinghe: It is probably worth noticing that the three elements $\mathrm{Ca}, \mathrm{Be}, \mathrm{Ti}$ share two properties in common: (1) they form strongly bound gaseous oxides, and (2) these gaseous oxides in turn can form highly refractory solids. It may be argued that much of the $\mathrm{Ca}, \mathrm{Be}$, Ti reaching the interstellar medium from stellar sources is locked away in the form of these highly refractory solids.

Herbig: There is no longer any evidence that Be is underabundant (see Herbig, $Z$. Astrophys. 68, 243,1968 ). As regards free gaseous $\mathrm{TiO}$ (and possibly $\mathrm{CaO}$ as well), resonance lines of these molecules are accessible, but despite specific search, have not been found in interstellar absorption. If ejection of solids by cool oxygen stars were common, I would think that some of these diatomic constituents of the atmospheres would be carried off into space along with the solids, and am therefore surprised that none is found.

Wickramasinghe: This would not be so if all the ambient oxide molecules were deposited as solid particles before they reached the interstellar medium. It may also be possible that these metallic oxides combined with $\mathrm{SiO}_{2}$ and actually condensed as silicates.

Greenberg: I should just like to mention another correlation (or anti-correlation) between interstellar $\mathrm{Ca}$ abundance and $4430 \AA$. It appears that where Ca abundance is relatively normal the 4430 $\AA$ is weak and this would seem to indicate that $4430 \AA$ (and perhaps the other diffuse lines) is due to $\mathrm{Ca}$ in some (as yet unknown) form either as bound in molecules or in the grains.

Herbig: Of course, it would be immensely satisfying if the carrier of the diffuse bands could also account for the $\mathrm{Ca}$ deficiency: e.g., if the $\mathrm{CaC}$ molecule could explain both.

Praderie: The ratio of heavy elements to calcium is known to be very different in normal stars as compared to Ap or Am stars. Concerning the origin of the peculiar stars and their relation with the neighbouring interstellar matter, have interstellar lines of heavy elements (e.g., rare earths) been ever detected?

Herbig: I have looked for interstellar lines of several rare earths in $\zeta$ Oph, without success (see Z. Astrophys. 68, 243, 1968). 
Note added November 1969: Since my remark at Lunteren regarding the CaC molecule as a constituent of the interstellar medium, I noted a paper by Espenhain et al. (Z. Astrophys. 61, 77, 1965), in which observations were reported of Ca vapor in contact with carbon in a King furnace at relatively high $\mathrm{H}_{2}$ pressures. They noted 11 unidentified bands in the 6256-6304 $\AA$ region, but neither their tracings nor those of Wurm and Meister (Z. Astrophys. 13, 25, 1936) show any hint of an absorption feature near $4430 \AA$. There is a diffuse feature at $4550 \AA$, found originally by Wurm and Meister, which is regarded as unidentified although lines of the $4^{3} P^{0}-4^{3} \mathrm{D}$ multiplet of $\mathrm{Ca}$ I occur near that wavelength. 